\title{
The Optimal Look-Ahead Policy for Admission to a Single Server System
}

\author{
W. M. NAWIJN \\ Twente University of Technology, Enschede, The Netherlands
}

(Received March 1982; accepted April 1984)

\begin{abstract}
This paper considers a service system with a single server, finite waiting room, and a renewal arrival process. Customers who arrive while the server is busy are lost. Upon completing service, the server chooses between two actions: either he immediately starts a new service, provided a customer is present, or he admits the newly arrived customer to the system, but delays service pending the next arrival, whereupon he again chooses between these two actions. This process continues until either the system is full or a new service is started. Once a service has been started, all customers who arrive while the server is busy are lost. We assume that at each decision epoch the server knows the arrival epoch of the first arriving customer. We show that there exists an optimal control-limit policy that minimizes the average expected idle time per customer served (equivalently, maximizes the average number of customers served per unit of time). The special case of Poisson arrivals leads to an explicit expression for this delay that generalizes exisiting results.
\end{abstract}

$\mathrm{T}$

HE MODEL considered in this paper originates from the following problem. Consider a work station, situated along a conveyor, comprised of a storage buffer of finite capacity and a machine served by an operator. Since the operator unloads the parts arriving on the conveyor and also performs the necessary operations on the parts, parts arriving during a service are lost. If the operator can always see the first oncoming part, the question arises as to which unloading policy will maximize the production rate of the work station.

Our model is a service system with one server and finite waiting room. Customers arrive according to an ordinary renewal process. Upon terminating a service, the server notes the time that elapses until the next arrival. We assume that he immediately detects this time with certainty. Having observed this time, the server can choose between two actions: either instantaneously start a new service, provided a customer is present, or delay service until the next arrival and admit the newly arriving customer to the system. In the latter case he notes the arrival epoch of the subsequent customer, which has just become known to him, and

Subject classification: 119 look-ahead policy for admission to single server system, 587 unloading policy for conveyor-serviced work station, 601 admission control to single server queue. 
again decides between delaying or starting a new service, and so on, until either the system is full or a new service is started. Once a service has been initiated, all customers who arrive while the server is busy are lost.

Our aim is to develop a stationary policy that minimizes the server's long-run average expected idle time per customer admitted to the system. In fact, this policy will maximize the average number of customers served per unit of time. Special cases of this model have been considered in the literature on "Conveyor-serviced Production Stations" (see Beightler and Crisp [1968], Matsui and Shingu [1978], Nawijn and De Jonge [1981], and Nawijn [1983]).

All these references used a priori policies without investigating the form of an optimal policy. In order to find an optimal policy, we will formulate the model as a Markov Decision Process and use an optimality theorem of Ross [1968].

The objective function to be minimized is the server's mean idle time per customer served or, in terms of the conveyor model mentioned above, the mean idle time per part produced, and this minimization problem yields the same optimal policy as the maximization of the average number of parts processed per unit of time (the production rate of the station).

\section{THE MATHEMATICAL MODEL AND SOME PRELIMINARIES}

The arrival process of customers is a renewal process with arrival rate $\lambda, 0<\lambda<\infty$, with interarrival time distribution $A(t)$. The service times are i.i.d. with distribution function $B(t)$, and are independent of the arrival process. We assume that $\beta=\int t d B(t)<\infty$. To minimize technical details, we also assume (i), that $A(t)$ and $B(t)$ are continuous on $[0, \infty)$ and (ii), that $0<A(t)<1$ for finite $t>0$, although the model can be solved for more general $A(\cdot)$ and $B(\cdot)$. Letting $s=\sup \{t: B(t)<1\} \leq \infty$, we will let $[0, s]$ denote the support of $B(\bullet)$. The maximum number of customers in the system is at most $K+1$. To describe the way in which customers are admitted to the system, we let $T_{2}(n)$ for $n \geq 1$ denote the termination epoch of the $n$th service time, and let $T_{1}(n ; j)$ for $n \geq 1$ and $j \geq 1$ denote the $j$ th arrival epoch after $T_{2}(n)$.

At epoch $T_{2}(n)$, the server can choose between two actions: either immediately start a new service, provided a customer is present, or delay service until the arrival epoch $T_{1}(n ; 1)$ and admit the arriving customer to the system. In the latter case, the server again either delays service until time $T_{1}(n ; 2)$, if the system is not full, or starts a new service, and so on. Once a new service has been started, say at epoch $T_{1}(n ; j)$, all customers arriving in the time interval $\left(T_{1}(n ; j), T_{2}(n+1)\right]$ are lost. Obviously, if at some epoch $T_{2}(n)$ the system is empty, the server will always wait for the next arrival. Let the sequence $\{T(n), n \geq 1\}$ denote the successive decision epochs. Moreover, let the state of the system at 
epoch $T(n)$ be defined by $\left(z_{n}, \tau_{n}\right)$, in which $z_{n}$ denotes the number of customers in the system at time $T(n)+0$ and $\tau_{n}$ denotes the time that elapses between $T(n)$ and the first arrival epoch after $T(n)$. Note that $\tau_{n}$ is a residual interarrival time if $T(n) \in\left\{T_{2}(n)\right\}$, or an ordinary interarrival time when $T(n)$ is an arrival epoch.

The state space of the process $\left\{\left(z_{n}, \tau_{n}\right), n \geq 1\right\}$ is $S=\{0,1, \ldots$, $\mathrm{K}+1\} x[0, \infty)$. For the initial state, we assume that $T(1)=0, z_{1}=i_{1}, \tau_{1}$ $=\tau_{1} ; i_{1} \in\{0,1, \cdots, K+1\}, 0<\tau_{1}<\infty$. We make the essential assumption that at all decision epochs $T(n)$ the server is able to observe when the next customer after $T(n)$ arrives, i.e., at epoch $T(n)+0$ the state $\left(z_{n}, \tau_{n}\right) \in S$ is known with certainty.

We wish to find a policy that minimizes the server's long-run average expected delay per served customer. To this end, we formulate the model as a discrete-time Markov Decision Process. Given that at some decision epoch the system is in state $(i, \tau) \in S$, the action $a(i, \tau)$ of the server is defined by

$$
\begin{aligned}
a(i, \tau) & =0, \text { if the server does not delay, } \\
& =1, \text { if the server delays. }
\end{aligned}
$$

Let $D(i, \tau)$ denote the set of admissible actions $\{a(i, \tau)\}$ in state $(i, \tau)$ $\in S$. Then, by assumption, we have

$$
D(i, \tau)= \begin{cases}\{1\} \quad \text { for } i=0, \quad \tau \geq 0 \\ \{0,1\} & \text { for } i=1,2, \ldots, K ; \quad \tau \geq 0 \\ \{0\} & \text { for } i=K+1, \quad \tau \geq 0\end{cases}
$$

Given the state $\left(z_{n}, \tau_{n}\right)=(i, \tau)$ and the action $a_{n}=a\left(z_{n}, \tau_{n}\right)=k$ taken at time $T(n)+0$, we can define the one-step transition probabilities by

$$
\operatorname{Pr}\left\{z_{n+1}=j, \tau_{n+1} \leq t \mid z_{n}=i, \tau_{n}=\tau, a_{n}=k\right\}=Q_{k}(i, \tau ; j, t),
$$

for $(i, \tau),(j, t) \in S$ and $k \in D(i, \tau)$.

$$
\begin{aligned}
& Q_{1}(i, \tau ; j, t)=\delta_{i+1, j} A(t) \\
& Q_{0}(i, \tau ; j, t)=\delta_{i-1, j} G(\tau, t),
\end{aligned}
$$

in which $\delta_{i, j}$ is Kronecker's symbol. The properties of the conditional probability distribution $G(\tau, t)$ are given in the following Lemma.

LEMMA 1.

(a) $G(\tau, t)=B(\tau)-B(\tau-t)+\int_{x=\tau}^{\infty} d B(x) \int_{\substack{x=x-\tau \\ u=x+t}}^{\infty}[1-A(x-\tau+t-$ $u)$ ] $d M(u), \tau, t \geq 0$, in which $M(u)=\sum_{n=1}^{\infty} A^{n *}(u)$, where $A^{n *}$ is the nth fold convolution of $A$ with itself.

(b) $H(\tau)=\tau-\int_{0}^{\infty} t G(\tau, d t)=\beta-(1 / \lambda)(1-B(\tau))-(1 / \lambda) \int_{\tau}^{\infty} M(t-$ $\tau) d B(t), \tau \geq 0$. The function $H(\tau)$ is continuous and monotone increasing on $[0, s)$, and $H(\tau) \rightarrow \beta(<\infty)$ when $\tau \rightarrow \infty$. 
(c) $\int_{0}^{\infty} G(\tau, t) d A(\tau)=G(0, t), t \geq 0$.

(d) $G\left(\tau_{1}, t\right)-G\left(\tau_{2}, t\right) \leq \lambda(1-A(t))\left\{H\left(\tau_{1}\right)-H\left(\tau_{2}\right)\right\}, \tau_{1}>\tau_{2} \geq 0$, $t \geq 0$.

(e) $\lim _{t \rightarrow \infty} G(\tau, t)=0$ for all finite $t \geq 0$.

Proof. Consider a decision epoch $T(n)$ at which the next arrival occurs after a time $\tau$ and at which the server has decided to start a new service. Then $\tau_{n+1}$ is the residual lifetime at time $x$ in a delayed renewal process, in which the first renewal occurs at time $\tau$, with renewal distribution $A$, where $x$ is randomized with respect to the distribution function $B(x)$.

Distinguishing between the two cases $x<\tau$ and $x \geq \tau$ and using a wellknown result for the distribution function of the residual lifetime (see, e.g., Cohen [1969], p. 109). We can easily derive part (a). The assertions (b), (c) and (e) are readily verified from (a). The proof of part (d) is straightforward, using integration by parts and the renewal equation.

The direct "cost" $w_{k}(i, \tau)$ incurred by making decision $k$ in state $(i, \tau)$ is defined by

$$
w_{k}(i, \tau)=\tau \delta_{k, 1},
$$

that is, the direct cost is the idle time of the server between two consecutive decision epochs.

Given an initial state $(i, \tau)$ and a policy $R$, let $a_{n}=a\left(z_{n}, \tau_{n}\right)$ be the decision taken at time $T(n)$ and let $w_{n}=w_{a_{n}}\left(z_{n}, \tau_{n}\right)$ denote the idle time of the server between $T(n)$ and $T(n+1)$.

Finally, let $C$ denote the class of all policies.

LEMMA 2. If $\tau<\infty$, then

(a) $\lim \sup _{n \rightarrow \infty}(1 / n) \sum_{j=1}^{n} E_{R}\left\{\tau_{j} \mid z_{1}=i, \tau_{1}=\tau\right\}<\infty$

(b) $\lim \sup _{n \rightarrow \infty}(1 / n) \sum_{j=1}^{n} E_{R}\left\{w_{j} \mid z_{1}=i, \tau_{1}=\tau\right\}={ }^{d} g_{R}(i, \tau)<\infty$ for any $i \in\{0,1, \cdots, K+1\}$ and any policy $R \in C$.

Proof. The proof is straightforward. It is based upon the observation that, in general, every decision epoch is preceded by at most $K+1$ uninterrupted services and the inequality

$$
\begin{aligned}
E_{R}\left\{\tau_{n} \mid \tau_{n-1}=t, a_{n-1}=\cdots=a_{j+1}=\right. & \left.0, a_{j}=1\right\} \\
& \leq t+\gamma, \quad n-1 \geq j+1 \geq 2,
\end{aligned}
$$

where

$$
\gamma=(1 / \lambda)+(1 / \lambda) \int_{0}^{\infty}\{M(t)-\lambda t\} d B(t)<\infty,
$$

which is finite since the mean service time is finite.

Recall that at a decision epoch $T(n)$, either a service completion occurs or a customer enters the system. Let these two types of epoch constitute, 
respectively, two time sequences, $\left\{T_{2}(n)\right\}$ and $\left\{T_{1}(n)\right\}$. Define $N_{j}(n)=$ $\max \left\{i\right.$ : $\left.T_{j}(i) \leq T(n), i=1,2, \cdots, n\right\}$ for $j=1,2$, i.e., $N_{j}(n)$ denotes the number of decision epochs of type $j$ up until time $T(n)$.

Lemma 3. Let $z_{1}=i$, and $\tau_{1}=\tau<\infty$. Then with probability one

$$
\lim _{n \rightarrow \infty} N_{j}(n) / n=1 / 2 \text { for } j=1,2
$$

for all $(i, \tau) \in S$ and any policy $R$ used.

Proof. Since $N_{1}(n)-N_{2}(n)$ gives the difference between the number of entered and departed customers during the time interval [0,T(n)], we have with probability one $0 \leq N_{1}(n)-N_{2}(n)+i \leq K+1$ for $n \geq 2$. Since, moreover, $N_{1}(n)+N_{2}(n)=n$ we obtain

$$
1-(1 / n) \leq(2 / n) N_{1}(n) \leq 1+(K+1-i) / n \quad \text { (a.s.), }
$$

from which the assertion follows.

Using Lemma 2 and Lemma 3, we can easily verify

LEMMA 4.

$$
\lim \sup _{n \rightarrow \infty} E_{R}\left\{\sum_{j=1}^{n} w_{j} / N_{2}(n) \mid z_{1}=i, \tau_{1}=\tau\right\}=2 g_{R}(i, \tau)<\infty .
$$

As optimality criterion, we wish to minimize the function $2 g_{R}(i, \tau)$. By Lemma 2 this function exists and is finite. If the ordinary limit in (1) exists, then $2 g_{R}(i, \tau)$ represents the long-run average expected idle time per customer served, when the initial state is $(i, \tau) \in S$ and policy $R$ is used.

A policy $R^{*}$ is optimal if $g_{R^{*}}(i, \tau) \leq g_{R}(i, \tau)$ for all $(i, \tau) \in S$ and any policy $R \in C$. In order to find an optimal policy and to prove its existence, we apply the following optimality theorem, which provides sufficient conditions for the existence of an optimal stationary deterministic policy. The theorem is a special case of a more general theorem due to Ross as adapted to the Markov Decision Process considered here.

Theorem 1. Suppose there exist a finite number $g$ and a set of Baire functions $\left\{f_{i}(\tau), i=0,1, \cdots, K+1\right\}$ defined for $\tau \geq 0$ that satisfy

(a) $\int_{0}^{\infty} f_{i}(t) d A(t)$ and $\int_{0}^{\infty} f_{i}(t) G(\tau, d t)$ exist and are finite, $i=0,1$, $\cdots, K+1$.

(b) $g+f_{0}(\tau)=\tau+\int_{0}^{\infty} f_{1}(t) d A(t)$

(c) $g+f_{i}(\tau)=\min \left\{\tau+\int_{0}^{\infty} f_{i+1}(t) d A(t), \int_{0}^{\infty} f_{i-1}(t) G(\tau, d t)\right\}, i=1,2$, $\cdots, K$

(d) $g+f_{K+1}(\tau)=\int_{0}^{\infty} f_{K}(t) G(\tau, d t)$

(e) $\lim _{n \rightarrow \infty}(1 / n) E_{R}\left\{f_{z_{n}}\left(\tau_{n}\right) \mid z_{1}=i, \tau_{1}=\tau\right\}=0$ for any $R \in C$ and all $(i, \tau) \in S$ with $\tau<\infty$.

Then, any stationary deterministic policy $R^{*}$ is optimal that, given a 
state $(i, \tau)$, prescribes an action $a(i, \tau)$ such that the right-hand side in (c) is minimized. Moreover, $g=g_{R^{*}}(i, \tau)$ for all $(i, \tau) \in S, \tau<\infty$, where the lim sup in Lemma 2 and (1) can be replaced by lim.

\section{AN OPTIMAL POLICY, ITS FORM AND EXISTENCE}

In this section we derive the form of an optimal stationary deterministic policy and prove its existence. To this end, we will deduce a solution of the extremal equations (b)-(d) in Theorem 1 and prove that the solution satisfies the conditions of the theorem. For notational convenience, let $f_{i}=\int_{0}^{\infty} f_{i}(t) d A(t)$ for $i=0,1, \ldots, K+1$.

Consider the functional equations, defined for $\tau \geq 0$,

$$
\begin{aligned}
f_{0}(\tau)+g & =\tau+f_{1} \\
f_{i}(\tau)+g & =\min \left\{\tau+f_{i+1}, \int_{0}^{\infty} f_{i-1}(t) G(\tau, d t)\right\}, \\
f_{K+1}(\tau)+g & =\int_{0}^{\infty} f_{K}(t) G(\tau, d t) .
\end{aligned}
$$

In order to show the existence of a solution to (2), let us introduce the functions

$$
h_{i}(\tau)=f_{i}(\tau)-\tau, \quad \tau \geq 0, \quad i=0,1, \cdots, K+1 .
$$

Then (2) is equivalent to

$$
\begin{aligned}
& h_{0}(\tau)+g=(1 / \lambda)+h_{1}, \\
& h_{i}(\tau)+g=\min \left\{\begin{array}{l}
(1 / \lambda)+h_{i+1}, \\
\int_{0}^{\infty} \mathrm{h}_{i-1}(t) G(\tau, d t)-H(\tau),
\end{array}\right.
\end{aligned}
$$

for $i=1,2, \cdots, K$,

$$
h_{K+1}(\tau)+g=\int_{0}^{\infty} h_{K}(t) G(\tau, d t)-H(\tau),
$$

where $h_{i}=\int_{0}^{\infty} h_{i}(\tau) d A(\tau), i=0,1, \cdots, K+1$.

Now observe that (4) belongs to a Markov Decision Process with the same state and action space as the original problem in which the costfunction $w_{k}(i, \tau)$ is replaced by

$$
\begin{aligned}
\bar{w}_{k}(i, \tau) & =-H(\tau), & & k=0 \\
& =1 / \lambda, & & k=1 .
\end{aligned}
$$

Since by Lemma 1 the function $H(\tau)$ is bounded, the costs in the modified 
problem are bounded. Now we can prove the existence of a solution to (4) along the lines of Ross. Let the functions $\left\{h_{i}{ }^{\alpha}(\tau), i=0,1, \cdots, K+1\right\}$ for $0<\alpha<1$ and the constant $g_{\alpha}$ provide the solution to the extremal equations that correspond to the discounted version of this modified Markov Decision Process. (This solution exists by a theorem of Blackwell [1965].) From these equations, it is possible to show that $\left\{h_{i}^{\alpha}(\tau)\right\}$ is a nondecreasing uniformly bounded equicontinuous family of functions. Then by Ross' Theorem 2, based on the Arzela-Aascoli theorem, there exist bounded continuous functions $\left\{h_{i}(\tau)\right\}$ and a constant $g$ satisfying (4), which by (3) guarantees a solution to the equations in (2).

REMARK 1. When we drop the assumption on the continuity of $A$, we can give the above proof using the monotonicity of the functions $\left\{h_{i}^{\alpha}(\tau)\right\}$ and by applying Helly's selection theorem instead of the Arzela-Ascoli theorem (see Nawijn).

Let us introduce the difference functions $V_{i}(\tau)$ for $i=0,1, \cdots, K$ and $\tau \geq 0$, defined by

$$
\begin{aligned}
& V_{0}(\tau)=0 \\
& V_{i}(\tau)=\tau+f_{i+1}-\int_{0}^{\infty} f_{i-1}(t) G(\tau, d t), \quad i=1,2, \cdots, K,
\end{aligned}
$$

in which the functions $\left\{f_{i}(\tau)\right\}$ satisfy relation (2) for some finite $g$.

Observe that

$$
f_{i}(\tau)+g=\tau+f_{i+1}-\max \left(0, V_{i}(\tau)\right),
$$

Since the construction of the solution proceeds in several steps, we first give a brief outline of the procedure.

First, we establish some properties of the functions $f_{i}(\tau)$ and $V_{i}(\tau)$. Then we prove (cf. Theorem 2) that if there exist an index $k \in$ $\{1,2, \cdots, K\}$ and a finite positive number $c_{k}$ such that $V_{i}(\tau) \leq 0$ for $i=$ $0,1, \cdots, k-1$ and $V_{k}(\tau) \leq 0$ for $0 \leq \tau \leq c_{k}$ and $V_{k}(\tau)>0$ for $\tau>c_{k}$, then there exist finite positive numbers $\left\{c_{i}\right\}$ for $i=k+1, \cdots, K$ such that $c_{k}>c_{k+1}>\ldots>c_{K}>0$ and $V_{i}(\tau)<0$ for $0 \leq \tau<c_{i}$, $V_{i}\left(c_{i}\right)=0, V_{i}(\tau)>0$ for $\tau>c_{i}, i=k, k+1, \cdots, K$.

Having obtained this property, we then prove (cf. Theorem 3 and Lemma 6) that for every solution of (2) such an index must exist. We then show that $k=1$ (cf. Theorem 4). The form of a solution to (2), given in Theorem 4, enables us to prove part (e) of Theorem 1. By then, we have fulfilled all conditions of Theorem 1, which then guarantees the existence of an optimal (critical value) policy.

We begin the construction of the solution by observing that if $\left\{f_{i}(\tau)\right\}$ satisfies (2) for some number $g$, then $\left\{f_{i}(\tau)+d\right\}$ is also a solution, where 
$d$ is some arbitrary constant. Hence, we can assume without a loss of generality that $f_{i}(\tau) \geq 0$ for $\tau \geq 0$ and $i=0,1, \cdots, K+1$.

LEMMA 5. If $V_{k-1}(\tau) \leq 0$ for $\tau \geq 0$, for some $k \in\{1,2, \cdots, K\}$, then

(a) $V_{k}(\tau)$ is continuous and bounded for $\tau \geq 0$, monotone increasing on $[0, s)$ and constant when $s<\tau<\infty$.

(b) $V_{k}(0)<0$.

Proof. Since $V_{k-1}(\tau) \leq 0$ for $\tau \geq 0$, we obtain from (6) and (7) that

$$
V_{k}(\tau)=\tau-\int_{0}^{\infty} t G(\tau, d t)+f_{k+1}-f_{k}+g
$$

Now, part (a) is an immediate consequence of Lemma 1, part b. To prove part (b), let us assume to the contrary that $V_{k}(0) \geq 0$. Consequently, $V_{k}(\tau) \geq 0$ for $\tau \geq 0$. We can easily verify from $V_{k}(\tau) \geq 0$ and $V_{k-1}(\tau) \leq$ 0 , for all $\tau \geq 0$, that

$$
2 g=\int_{0}^{\infty} t G(0, d t)
$$

Moreover, since $V_{k}(\tau) \geq 0$ and $f_{k+1}(\tau)+g \leq \int_{0}^{\infty} f_{k}(t) G(\tau, d t)$, it follows that

$$
g+f_{k+1} \leq \int_{0}^{\infty} f_{k}(t) G(0, d t) \leq \int_{0}^{\infty} t G(0, d t)+f_{k+1}-g .
$$

Hence, by (9)

$$
\int_{0}^{\infty} f_{k}(t) G(0, d t)=f_{k+1}+g .
$$

Now from (8) and (10) we have, in view of $V_{k-1}(\tau) \leq 0$ and $V_{k}(\tau) \geq 0$,

$$
\begin{aligned}
V_{k}(0) & =\int_{0}^{\infty} f_{k}(t) G(0, d t)-f_{k}-\int_{0}^{\infty} t G(0, d t) \\
& =\int_{0}^{\infty} \int_{0}^{\infty} f_{k-1}(u) G(u, d t) G(0, d t)-f_{k}-g-\int_{0}^{\infty} t G(0, d t) \\
& =\int_{0}^{\infty} \int_{0}^{\infty} u G(t, d u) G(0, d t)-4 g \\
& =-\int_{0}^{\infty}\{H(t)-H(0)\} G(0, d t) .
\end{aligned}
$$

The latter quantity, however, is strictly negative, as can be verified from Lemma $1(\mathrm{~b})$, which contradicts the hypothesis $V_{k}(0) \geq 0$. Hence, we necessarily have $V_{k}(0)<0$ and the proof is complete. 
This lemma leads to the following corollary:

Corollary 1. $2 g<\int_{0}^{\infty} t G(0, d t)=(1 / \lambda)-\beta+(1 / \lambda) \int_{0}^{\infty} M(t) d B(t)$.

We now turn to the key result on which most of the remaining results in this paper rest. It provides the basis for the form of the optimal policy.

THEOREM 2. If there exist an index $k \in\{1,2, \cdots, K\}$ and a finite positive number $c_{k}$ such that $V_{k}(\tau)<0$ for $0 \leq \tau<c_{k}, V_{k}\left(c_{k}\right)=0, V_{k}(\tau)>0$ for $\tau>c_{k}$ and $V_{j}(\tau) \leq 0$ for $\tau \geq 0, j=0,1, \cdots, k-1$, then there exist finite positive numbers $\left\{c_{i}\right\}$ such that for $i=k, \cdots, K$ :

(a) $V_{i}(\tau)<0$ for $0 \leq \tau<c_{i}, V_{i}\left(c_{i}\right)=0, V_{i}(\tau)>0$ for $\tau>c_{i}$;

(b) $c_{i-1}>c_{i}>0$ and $c_{i}<s$;

(c) $\int_{0}^{c_{i}}(1-A(t)) d V_{i}(t)=(1 / \lambda)-\int_{c_{i}}^{\infty} V_{i}(t) d A(t)=f_{i}+g-f_{i+1}$.

Proof. Since $V_{k-1}(\tau) \leq 0$ for $\tau \geq 0, V_{k}(\tau)$ is continuous and bounded for $\tau \geq 0$ and, moreover, strictly increasing on $[0, s)$ by Lemma 5. Part (a) is trivially true by assumption for $i=k$. The first part of (b) for $i=k$ is true when $c_{k-1}$ is interpreted as infinity. To prove the second part of (b) for $i=k$, note that when $s=\infty$ we trivially have $c_{k}<s$. When $s<\infty$ we have $V_{k}(\tau)=V_{k}(s)=\lim _{\tau \rightarrow \infty} V(\tau)>0$ for $\tau \geq s$, which is easily verified from (cf. (8)).

$$
V_{k}(\tau)=\tau-\int_{0}^{\infty} t G(\tau, d t)+f_{k+1}-f_{k}+g, \quad \tau \geq 0,
$$

using Lemma 1, part (b). Hence, since $V\left(c_{k}\right)=0, c_{k}<s$.

To prove part (c) for $i=k$, observe first that

$$
\int_{0}^{\infty}(1-A(t)) d V_{k}(t)=-V_{k}(0)+\int_{0}^{\infty} V_{k}(t) d A(t)
$$

noting that $V_{k}(\infty)<\infty$ and $A(0)=0$. Hence, using (11) we have

$$
\begin{aligned}
\int_{0}^{\infty}(1 & -A(t)) d V_{k}(t) \\
= & \int_{0}^{\infty} t G(0, d t)-f_{k+1}+f_{k}-g \\
& +\int_{0}^{\infty}\left\{\tau-\int_{0}^{\infty} t G(\tau, d t)+f_{k+1}-f_{k}+g\right\} d A(\tau)=1 / \lambda,
\end{aligned}
$$

applying Fubini's theorem and Lemma 1 part (c). Integrating (7) for 
$i=k$ with respect to $A(\tau)$ yields

$$
\begin{aligned}
f_{k}+g & =(1 / \lambda)+f_{k+1}-\int_{0}^{\infty} \max \left(0, V_{k}(t)\right) d A(t) \\
& =(1 / \lambda)+f_{k+1}-\int_{c_{k}}^{\infty} V_{k}(t) d A(t),
\end{aligned}
$$

since $V_{k}(t) \leq 0$ for $t \leq c_{k}$ and $V_{k}(t)>0$ for $t>c_{k}$, by hypothesis. By partial integration, we obtain

$$
\int_{c_{k}}^{\infty}(1-A(t)) d V_{k}(t)=\int_{c_{k}}^{\infty} V_{k}(t) d A(t),
$$

since $V_{k}\left(c_{k}\right)=0$ and $V_{k}(\infty)<\infty$. The proof of part (c) for $i=k$ now follows by combining the relations (12), (13) and (14).

In the sequel we will use the following recurrence relation between $V_{i+1}(\tau)$ and $V_{i}(\tau)$

$$
\begin{aligned}
V_{i+1}(\tau)= & \tau-\int_{0}^{\infty} t G(\tau, d t)+\int_{0}^{\infty} \max \left(0, V_{i}(t)\right) G(\tau, d t) \\
& +f_{i+2}-f_{i+1}+g, \quad i=0,1, \cdots, K-1
\end{aligned}
$$

which is easily verified by substituting (7) in (6).

Since $V_{k}(\tau)>0$ if and only if $\tau>c_{k}$, (15) implies that

$$
\begin{aligned}
V_{k+1}(\tau)= & \tau-\int_{0}^{\infty} t G(\tau, d t)+\int_{c_{k}}^{\infty} V_{k}(t) G(\tau, d t) \\
& +f_{k+2}-f_{k+1}+g .
\end{aligned}
$$

We now wish to prove that $V_{k+1}(\tau)>V_{k}(\tau)$ for $\tau \geq 0$. Consider the difference

$$
V_{k+1}(\tau)-V_{k}(\tau)=\int_{c_{k}}^{\infty} V_{k}(t) G(\tau, d t)+f_{k+2}-2 f_{k+1}+f_{k}
$$

which is easily found from (11) and (16). Since the integral in (17) is positive, we wish to show that $f_{k+2}-2 f_{k+1}+f_{k} \geq 0$, in order that $V_{k+1}(\tau)>V_{k}(\tau), \tau \geq 0$. By part (c) for $i=k$, we have

$$
f_{k}-f_{k+1}+g=(1 / \lambda)-\int_{c_{k}}^{\infty} V_{k}(t) d A(t)
$$

Moreover, observe from (2) that

$$
f_{k+1}(\tau)+g \leq\left\{\begin{array}{lr}
\tau+f_{k+2}, & 0 \leq \tau<c_{k} \\
\int_{0}^{\infty} f_{k}(t) G(\tau, d t), \quad \tau>c_{k}
\end{array}\right.
$$


Integrating both sides with respect to $A(\tau)$ and using (6), we obtain

$$
f_{k+1}-f_{k+2}+g \leq(1 / \lambda)-\int_{c_{k}}^{\infty} V_{k+1}(t) d A(t) .
$$

Hence, in view of (18) and (19)

$$
f_{k+2}-2 f_{k+1}+f_{k} \geq \int_{c_{k}}^{\infty}\left\{V_{k+1}(t)-V_{k}(t)\right\} d A(t) .
$$

Inserting (11) and (16) in the right-hand side, we obtain, after rearranging terms,

$$
A\left(c_{k}\right)\left\{f_{k+2}-2 f_{k+1}+f_{k}\right\} \geq \int_{c_{k}}^{\infty} \int_{c_{k}}^{\infty} V_{k}(t) G(\tau, d t) d A(\tau)>0,
$$

hence

$$
f_{k+2}-2 f_{k+1}+f_{k}>0
$$

and, consequently, in view of (17),

$$
V_{k+1}(\tau)>V_{k}(\tau), \quad \tau \geq 0 .
$$

We now show inductively that for $i=k, k+1, \cdots, K$

(i) $V_{i}(\tau)$ is continuous and bounded for $\tau \geq 0$ and increasing on $[0, s)$

(ii) $V_{i}(0)<0$;

(iii) $V_{i}(\tau)>V_{i-1}(\tau), \tau \geq 0(i \geq k+1)$;

(iv) $\int_{0}^{c_{i}}(1-A(t)) d V_{i}(t)=(1 / \lambda)-\int_{c_{i}}^{\infty} V_{i}(t) d A(t)=f_{i}+g-f_{i-1}$.

Observe that the assertions (i)-(iv) together with the fact that $V_{k}\left(c_{k}\right)=$ 0 and $V_{k}(\tau)>0$ for $\tau>c_{k}$ imply the assertions (a), (b) and (c) of the theorem. The assertions (i)-(iv) have been proved for $i=k$. Assuming that we have proved the assertions (i)-(iv) for $i=k, k+1, \cdots$, $n(n<K)$, we advance the induction to $n+1$. The proof is given below.

(i) Since $V_{n}(\tau) \leq 0$ for $\tau \leq c_{n}$, we have from (15)

$$
\begin{aligned}
V_{n+1}(\tau)= & \tau-\int_{0}^{\infty} t G(\tau, d t)+\int_{c_{n}}^{\infty} V_{n}(t) G(\tau, d t) \\
& +f_{n+2}-f_{n+1}+g .
\end{aligned}
$$

This relation can be written, using integration by parts, as

$$
\begin{aligned}
V_{n+1}(\tau)= & \tau-\int_{0}^{\infty} t G(\tau, d t)-\int_{c_{n}}^{\infty} G(\tau, t) d V_{n}(t) \\
& +f_{n+2}-f_{n+1}+g+V_{n}(\infty),
\end{aligned}
$$

since $V_{n}\left(c_{n}\right)=0$ and $V_{n}(\infty)<\infty$. 
From Lemma 1, the uniform convergence of the second integral, and the continuity of $A$ and $B$, we can see that $V_{n+1}(\tau)$ is continuous for $\tau \geq 0$. Moreover, $V_{n+1}(\tau)$ is bounded for $\tau \geq 0$ in view of Lemma 1 and the finiteness of the second integral. Now consider the difference $V_{n+1}\left(\tau_{1}\right)$ - $V_{n+1}\left(\tau_{2}\right)$ for $\tau_{1}>\tau_{2} \geq 0$. Using (24) and part (d) of Lemma 1, we can verify

$$
\begin{aligned}
V_{n+1}\left(\tau_{1}\right)- & V_{n+1}\left(\tau_{2}\right) \\
& \geq\left\{1 / \lambda-\int_{c_{n}}^{\infty}(1-A(t)) d V_{n}(t)\right\}\left\{H\left(\tau_{1}\right)-H\left(\tau_{2}\right)\right\} .
\end{aligned}
$$

Using partial integration and (iv) for $i=n$, we can write the first factor on the right as

$$
\int_{0}^{c_{n}}(1-A(t)) d V_{n}(t)>0,
$$

since $s>c_{n}>0$ and $V_{n}(t)$ is strictly increasing on $[0, s)$. The second factor is positive since the function $H(\tau)$ is increasing on $[0, s)$ in view of Lemma 1, part (b). Hence, $V_{n+1}(\tau)$ is increasing on $[0, s)$, which finally proves (i) for $i=n+1$. Further, note that $V_{n+1}(\tau)$ is nondecreasing for $\tau>s$ in case $s<\infty$.

(iii) To prove assumption (iii) for $i=n+1$, observe that

$$
\begin{aligned}
V_{n+1}(\tau)-V_{n}(\tau)= & \int_{c_{n}}^{\infty} V_{n}(t) G(\tau, d t)-\int_{c_{n-1}}^{\infty} V_{n-1}(t) G(\tau, d t) \\
& +f_{n+2}-2 f_{n+1}+f_{n} .
\end{aligned}
$$

By the same argument as that used in deriving (20), we obtain

$$
\begin{aligned}
A\left(c_{n}\right) & \left\{f_{n+2}-2 f_{n+1}+f_{n}\right\} \\
& \geq \int_{c_{n}}^{\infty} d A(\tau)\left\{\int_{c_{n}}^{\infty} V_{n}(t) G(\tau, d t)-\int_{c_{n-1}}^{\infty} V_{n-1}(t) G(\tau, d t)\right\} .
\end{aligned}
$$

Hence, $f_{n+2}-2 f_{n+1}+f_{n}>0$ since $c_{n-1}>c_{n}>0$ and $V_{n}(t)>V_{n-1}(t)$ for $t \geq 0$. Consequently, for the same reason, (26) implies that

$$
V_{n+1}(\tau)>V_{n}(\tau), \quad \tau \geq 0,
$$

which proves (iii) for $i=n+1$.

(ii) From (23) we obtain

$$
\begin{aligned}
V_{n+1}(0)= & -\int_{0}^{\infty} t G(0, d t)+\int_{c_{n}}^{\infty} V_{n}(t) G(0, d t) \\
& +f_{n+2}-f_{n+1}+g .
\end{aligned}
$$


Moreover, from (2), Lemma 1, and Fubini's theorem, we have

$$
\begin{aligned}
f_{n+2}+g & \leq \int_{0}^{\infty} f_{n+1}(t) G(0, d t) \\
& =\int_{0}^{\infty} t G(0, d t)-\int_{0}^{\infty} \max \left(0, V_{n+1}(t)\right) G(0, d t)+f_{n+2}-g .
\end{aligned}
$$

Applying this inequality to (29), we obtain

$$
\begin{aligned}
V_{n+1}(0) \leq \int_{c_{n}}^{\infty}\left\{V_{n}(t)\right. & \left.-V_{n+1}(t)\right\} G(0, d t) \\
& -\int_{0}^{c_{n}} \max \left(0, V_{n+1}(t)\right) G(0, d t)+f_{n+2}-f_{n+1}-g,
\end{aligned}
$$

noting that $V_{n+1}(t)>V_{n}(t) \geq 0$ for $t \geq c_{n}$ in view of (28), which for the same reason implies that

$$
V_{n+1}(0)<f_{n+2}-f_{n+1}-g .
$$

Now, suppose to the contrary, that $V_{n+1}(0) \geq 0$. Then by the usual argument, (7) and the fact that $V_{n+1}(\tau) \geq 0$, since $V_{n+1}(0) \geq 0$, implies that

$$
f_{n+1}-f_{n+2}+g=(1 / \lambda)-\int_{0}^{\infty} V_{n+1}(\tau) d A(\tau)
$$

Moreover,

$$
\begin{aligned}
\int_{0}^{\infty} V_{n+1}(\tau) d A(\tau) & =V_{n+1}(0)+\int_{0}^{\infty}(1-A(\tau)) d V_{n+1}(\tau) \\
& =V_{n+1}(0)+1 / \lambda
\end{aligned}
$$

by the same argument as that used for $i=k$ (cf. (12)). Combining (31) and (32), we obtain $V_{n+1}(0)=f_{n+2}-f_{n+1}-g$, which contradicts (30). Hence we must have $V_{n+1}(0)<0$, which proves (ii) for $i=n+1$.

Having advanced the inductive step to $i=n+1$, we know that the assumptions (i)-(iii) hold for $i=k, k+1, \cdots, K$. Finally, assumption (iv) can be established in exactly the same way as was done for $i=k$ (cf. (12), (13) and (14)). The proof is complete.

In view of Lemma 5 and the fact that $V_{0}(\tau)=0$, we either have $V_{1}(\tau)$ $\leq 0$ for $\tau \geq 0$ or $V_{1}(\tau) \leq 0$ for $0 \leq \tau \leq c_{1}<s$ and $V_{1}(\tau)>0$ for $\tau>c_{1}$. In the latter case, we can apply Theorem 2 , whereas in the former case we can again apply Lemma 5 . Repeating the argument results in the following theorem.

Theorem 3. If the functions $\left\{f_{i}(\tau), \tau \geq 0 ; i=0,1, \cdots, K+1\right\}$ satisfy (2) 
for some finite number $g$ then for some integer $k \in\{0,1, \cdots, K\}$

$$
f_{i}(\tau)+g=\tau+f_{i+1}, \quad \tau \geq 0, \text { for } i=0,1, \cdots, k,
$$

and

$$
f_{i}(\tau)+g= \begin{cases}\tau+f_{i+1}, & 0 \leq \tau \leq c_{i}, \\ \int_{0}^{\infty} f_{i-1}(t) G(\tau, d t), & \tau>c_{i},\end{cases}
$$

for $i=k+1, \cdots, K$, where $s>c_{k}>c_{k+1}>\cdots>c_{K}>0$.

Lemma 6. Under the conditions of Theorem 3, there exist an index $k \in\{1,2, \cdots, K\}$ and a corresponding finite positive number $c_{k}$ such that $V_{k}(\tau)<0$ for $0 \leq \tau<c_{k}, V_{k}\left(c_{k}\right)=0$, and $V_{k}(\tau)>0$ for $\tau>c_{k}$.

Proof. Suppose to the contrary that such an index does not exist. Then, according to Theorem 3 ,

$$
f_{i}(\tau)+g=\tau+f_{i+1}, \quad i=0,1, \cdots, K .
$$

Hence, from (2) and (33) we find by the usual argument that

$$
f_{K+1}+g=\int_{0}^{\infty}\left\{\tau+f_{K+1}-g\right\} G(0, d t) .
$$

However, this result yields $2 g=\int_{0}^{\infty} t G(0, d t)$, which contradicts Corollary 1. Consequently, (33) is impossible and in view of Theorem 3 there must be an index $k \in\{1,2, \cdots, K\}$ such that $V_{k}(\tau)>0$ for $\tau>c_{k}>0$, and $V_{i}(\tau) \leq 0$ for $\tau \geq 0(i=0,1, \cdots, k-1)$. The proof is complete.

We are now in a position to state the form of a solution of the extremal equations (2).

Theorem 4. If the functions $\left\{f_{i}(\tau), i=0,1, \cdots, K+1\right\}$ satisfy the extremal equations (2) for some finite number $g$, then

$$
\begin{aligned}
& f_{0}(\tau)+g=\tau+f_{i}, \quad \tau \geq 0, \\
& f_{i}(\tau)+g=\left\{\begin{array}{ll}
\tau+f_{i+1}, & 0 \leq \tau \leq c_{i}, \\
\int_{0}^{\infty} f_{i-1}(t) G(\tau, d t), & \tau>c_{i},
\end{array} \quad(i=1,2, \cdots, K),\right. \\
& f_{K+1}(\tau)+g=\int_{0}^{\infty} f_{K}(t) G(\tau, d t), \quad \tau \geq 0, \\
& \text { and } \\
& 2 g=(1 / \lambda)-\beta+\int_{c_{1}}^{\infty} A(t) d H(t), \\
& \infty \geq s>c_{1}>c_{2}>\ldots>c_{K}>0 .
\end{aligned}
$$

Moreover, $V_{i}\left(c_{i}\right)=0(c f .(6))$ and $f_{i}+g=(1 / \lambda)+f_{i+1}-\int_{c_{i}}^{\infty} V_{i}(\tau) d A(\tau)$, $i=1,2, \cdots, K$.

Proof. According to Lemma 6, there is an index $k \in\{1,2, \cdots, K\}$ such 
that $V_{i}(\tau) \leq 0$ for $i=1,2, \cdots, k-1$ and $V_{k}(\tau)<0$ for $0 \leq \tau<c_{k}, V_{k}\left(c_{k}\right)$ $=0, V_{k}(\tau)>0$ for $\tau>c_{k}$. In view of Theorem 2, we have $c_{k}<s$. When $k=1$, the theorem follows directly from Theorem 2 . To prove (35) when $k=1$, note that in this case $V_{1}(\tau)=H(\tau)+f_{2}-f_{1}+g$, so that $V_{1}(\infty)=$ $\beta+f_{2}-f_{1}+g$. Combining this result with

leads simply to (35).

$$
\begin{aligned}
f_{1}+g & =(1 / \lambda)+f_{2}-\int_{c_{1}}^{\infty} V_{1}(\tau) d A(\tau) \\
& =(1 / \lambda)+f_{2}-V_{1}(\infty)+\int_{c_{1}}^{\infty} A(\tau) d V_{1}(\tau),
\end{aligned}
$$

Now we will prove that $k=1$ is the only index for which Lemma 6 can be true. Suppose to the contrary that $k \geq 2$. Since $V_{k-2}(\tau) \leq 0$ and $f_{k-1}+$ $g=(1 / \lambda)+f_{k}$, it is easy to verify from (7) that

$$
V_{k-1}(\tau)=H(\tau)+2 g-(1 / \lambda)
$$

and hence

$$
V_{k-1}(\infty)=\beta-(1 / \lambda)+2 g \text {. }
$$

Analogously to (35), we find

$$
2 g-(1 / \lambda)+\beta=\int_{c_{k}}^{\infty} A(t) d V_{k}(t)>0,
$$

since $c_{k}<s$. Hence, $V_{k-1}(\infty)>0$, which contradicts the hypothesis that $V_{k-1}(\tau) \leq 0$ for $\tau \geq 0$. Consequently, $k \geq 2$ is impossible. The proof is complete.

COROLlaRY 2. Under the conditions of Theorem 4

$$
(1 / \lambda)-\beta<2 g<(1 / \lambda)-\beta+(1 / \lambda) \int_{0}^{\infty} M(t) d B(t) .
$$

Proof. This result follows immediately from (35) and (36).

Theorem 5. There exist continuous functions $\left\{f_{i}(\tau), i=0,1, \cdots, K+1\right\}$ and a finite constant $g$ satisfying the extremal equations (2). Moreover,

$$
\lim _{n \rightarrow \infty}(1 / n) E_{R}\left\{f_{z_{n}}\left(\tau_{n}\right) \mid z_{1}=i, \tau_{1}=\tau\right\}=0, \text { for all }(i, \tau) \in S
$$

and any policy $R \in C$, with $\tau<\infty$.

Proof. From Theorem 4, we deduce that

$$
\begin{aligned}
E_{R}\left\{f_{z_{n}}\left(\tau_{n}\right) \mid z_{1}=\right. & \left.i, \tau_{1}=\tau\right\} \\
\leq & \sum_{j=0}^{K} f_{j+1} \operatorname{Pr}_{R}\left\{z_{n}=j \mid z_{1}=i, \tau_{1}=\tau\right\}-g \\
& +E_{R}\left\{\tau_{n} \mid z_{1}=i, \tau_{1}=\tau\right\} \\
& +\left\{(1 / \lambda)+f_{K+1}-g\right\} \operatorname{Pr}_{R}\left\{z_{n}=K+1 \mid z_{1}=i, \tau_{1}=\tau\right\} .
\end{aligned}
$$

Hence, by applying Lemma 2, we can easily see that (38) holds. 
Now we finally have

THEOREM 6. There exists a unique optimal stationary deterministic policy $R^{*}$ such that

$$
\begin{aligned}
& a_{R^{*}}(i, \tau)=1 \text { if } 0 \leq \tau \leq c_{i} \\
& =0 \text { if } \tau>c_{i} \text {, }
\end{aligned}
$$

where the critical numbers $\left\{c_{i}\right\}$ for $i=1,2, \cdots, K$ satisfies $s>c_{1}>c_{2}>$ $\cdots>c_{K}>0$.

Proof. Clearly in view of Theorem 5, all the premises of Theorem 1 are fulfilled. Hence, there exists a stationary deterministic policy $R^{*}$ such that $g=g_{R^{*}}(i, \tau)=\min _{R} g_{R}(i, \tau)$ for all $(i, \tau) \in S$, with $\tau<\infty$. Since any solution of (2) is of the form given in Theorem 4, the optimal policy $R^{*}$ is a control-limit policy satisfying (39). It remains to prove that the critical numbers $\left\{c_{i}\right\}$ are unique.

We can verify from Theorem 2 and relation (15) that

$$
f_{n}+g=f_{n+1}+(1 / \lambda)-V_{n}(\infty)+\int_{c_{n}}^{\infty} A(\tau) d V_{n}(\tau)
$$

and

$$
V_{n}(\infty)=\beta+f_{n+1}-f_{n}+g+V_{n-1}(\infty) \text {. }
$$

Hence,

$$
2 g+\beta-(1 / \lambda)+V_{n-1}(\infty)-\int_{c_{n}}^{\infty} A(\tau) d V_{n}(\tau)=0, \quad n=1,2, \cdots, K,
$$

i.e., $c_{n}$ is a zero of the function $F_{n}(x)$ defined by

$$
F_{n}(x)=2 g+\beta-(1 / \lambda)+V_{n-1}(\infty)-\int_{x}^{\infty} A(\tau) d V_{n}(\tau), \quad x \geq 0 .
$$

In view of the properties of $V_{n}(\cdot), F_{n}(x)$ is continuous and increasing on $[0, s)$. Taking $n=1$ and noting that $V_{0}(\tau)=0$, we obtain

$$
F_{1}(x)=2 g+\beta-(1 / \lambda)-\int_{x}^{\infty} A(\tau) d H(\tau), \quad x \geq 0 .
$$

Since $g_{R^{*}}(i, \tau)=g$ for any optimal policy $R^{*}$, the critical number $c_{1}$ must be unique. Now suppose that $c_{1}, \cdots, c_{n-1}(n \leq K)$ are unique. Observing that $F_{n}(x)$ depends only on $c_{1}, \cdots, c_{n-1}$, we can see that the only root $c_{n}$ of $F_{n}(x)=0$ is also unique, and the proof follows by induction.

REMARK 2. We have focussed our attention on the minimization of the long-run average expected idle time per customer served, leading to the extremal equations (2). The more natural optimality criterion is the number of customers served per unit time. The extremal equations for the corre- 
sponding semi-Markov Decision Process read

$$
\begin{aligned}
f_{0}(\tau) & =1+f_{1}-\tilde{g} \tau \\
f_{1}(\tau) & =\max \left\{\begin{array}{l}
1+f_{i+1}-\tilde{g} \tau \\
\int_{0}^{\infty} f_{i-1}(t) G(\tau, d t)-\tilde{g} \beta
\end{array} \quad(i=1,2, \ldots, K)\right. \\
f_{K+1}(\tau) & =\int_{0}^{\infty} f_{K}(t) G(\tau, d t)-\tilde{g} \beta
\end{aligned}
$$

and it is possible to show that both formulations yield the same maximal production rate.

\section{POISSON ARRIVALS}

In this section we will apply our results to the case of a Poisson arrival process.

Since the renewal function is given by $M(t)=\lambda t$ for $t \geq 0$, Lemma 1 , part (a) implies that

$$
G(\tau, t)=B(\tau)-B(\tau-t)+A(t)(1-B(\tau)), \quad \tau \geq 0, \quad t \geq 0 .
$$

Inserting this result into Theorem 4 we can easily show that

$$
f_{i}(\tau)+g= \begin{cases}\tau+f_{i+1} & 0 \leq \tau \leq c_{i}, \\ (1-B(\tau)) f_{i-1}+\int_{0}^{\tau} f_{i-1}(\tau-t) d B(t), & \tau>c_{i},\end{cases}
$$

for $i=1,2, \cdots, K$.

Using the fact that

$$
f_{i}(\tau)+g=\tau+f_{i+1} \quad \text { for } \quad 0 \leq \tau \leq c_{i}, \quad i=1,2, \cdots, K
$$

we obtain from (6) and (40) that

$$
\begin{aligned}
V_{i}(\tau)= & \int_{0}^{\tau}(1-B(u)) d u \\
& -\left(f_{i-1}-f_{i}+g\right)(1-B(\tau))+f_{i+1}-f_{i}+g, \\
& 0 \leq \tau \leq c_{i-1} .
\end{aligned}
$$

By straightforward calculations, using (41) and noting that $V_{i}\left(c_{i}\right)=0$, we can verify from Theorem 2 part (c) that

$$
\begin{aligned}
f_{i}- & f_{i+1} \\
= & \left(1-\Phi_{i}\right)\left(f_{i-1}-f_{i}\right) / A_{i}-\left(1-A_{i}\right) \int_{0}^{c_{i}}(1-B(u)) d u / A_{i} \\
& +(1 / \lambda)\left\{1+\left(1-A_{i}\right) / A_{i}-\left(1-\Phi_{i}\right) / A_{i}\right\} \\
& -g\left\{1+2\left(1-A_{i}\right) / A_{i}-\left(1-\Phi_{i}\right) / A_{i}\right\},
\end{aligned}
$$


in which

$$
\begin{array}{r}
A_{i}=1-e^{-\lambda c_{i}} \text { and } \Phi_{i}=\int_{0}^{c_{i}}(1-B(u)) d A(u), \\
i=1,2, \cdots, K .
\end{array}
$$

Moreover, from (2) we have

$$
f_{0}-f_{1}=(1 / \lambda)-g \text { and } f_{K}-f_{K+1}=g,
$$

since $G(0, t)=A(t)$.

Solving the recurrence relations (42) and (43) for g, we obtain

$$
2 g=(1 / \lambda)-\sum_{n=0}^{K-1} \pi_{n} \int_{0}^{c_{n+1}}(1-B(u)) d u,
$$

where

$$
\begin{array}{r}
\pi_{n}=\left(1-A_{n+1}\right) \prod_{k=n+2}^{K}\left\{\left(1-\Phi_{k}\right) / A_{k}\right\} / A_{n+1}, \\
n=0,1, \ldots, K-1
\end{array}
$$

and $\pi_{K}$ can be found from $\sum_{n=0}^{K} \pi_{n}=1$. (The empty product in case $n=$ $K-1$ is to be interpreted as having value one).

Observe that (44) and (45) hold for every deterministic critical number policy with $c_{1} \geq c_{2} \geq \cdots \geq c_{K}>0$. Relation (44) generalizes results of Beightler and Crisp and Nawijn and De Jonge. Beightler and Crisp considered the case $c_{1}=c_{2}=\ldots=c_{K}=c$ and $B(c)=0$ (in fact, they considered a Bernoulli arrival process). Nawijn and De Jonge derived a special case of (44); in particular, they assumed that $B\left(c_{i}\right)=0$ for $i=1$, $2, \cdots, K$, in which case the process $\left\{z_{n}, n \geq 0\right\}$ is an ergodic Markov chain.

For the optimal policy, the critical numbers $\left\{c_{n}\right\}$ satisfy the simultaneous equations (42), (43) and $V_{n}\left(c_{n}\right)=0$, i.e.,

$$
\begin{array}{r}
\int_{0}^{c_{n}}(1-B(u)) d u-\left(f_{n-1}-f_{n}+g\right)\left(1-B\left(c_{n}\right)\right)+f_{n+1}-f_{n}+g=0, \\
n=1,2, \cdots, K .
\end{array}
$$

In the special case of large service times, (46) becomes

$$
c_{n}=f_{n-1}-f_{n+1}, \quad n=1,2, \cdots, K .
$$

and (42) becomes

$$
A_{n}\left(f_{n}-f_{n+1}\right)=\left(1-A_{n}\right)\left(f_{n-1}-f_{n}\right)-\left(1-A_{n}\right) c_{n}+A_{n} / \lambda-g .
$$

From (43), (47) and (48), we can verify that the critical numbers satisfy the set of equations

$$
\lambda c_{n}=\lambda c_{n+1}+e^{-\lambda c_{n+1}}-e^{-\lambda c_{n-1}}, \quad n=1,2, \cdots, K,
$$

with $c_{0}=\infty$ and $c_{K+1}=0$. 


\section{REFERENCES}

Beightler, C. S., AND R. M. CrisP. 1968. A Discrete-Time Queueing Analysis of Conveyor-Serviced Production Stations. Opns. Res. 16, 986-1001.

Blackwell, D. 1965. Discounted Dynamic Programming, Ann. Math. Statist. 36, 226-235.

Cohen, J. W. 1969. The Single Server Queue. North-Holland, Amsterdam.

Matsui, M., AND T. Shingu. 1978. A Queueing Analysis of Conveyor-serviced Production Station and the Optimal Range Strategy. AIIE Trans. 10(1), 89-99.

NawiJn, W. M. 1983. Stochastic Conveyor Systems, unpublished Ph.D. dissertation, Twente University of Technology, Enschede, the Netherlands.

NawiJn, W. M., AND R. De Jonge. 1981. The Analysis of Conveyor-serviced Production Station. Eur. J. Opnl. Res. 6, 67-74.

Ross, S. M. 1968. Arbitrary State Markovian Decision Processes. Ann. Math. Statist. 39(6), 2118-2122. 